This is the final peer-reviewed accepted manuscript of:

D. Fabiani, G. Mazzanti, S. V. Suraci and B. Diban

"Preliminary Development and Application of a Stress-Strength Model for Reliability Estimation of Aged LV Cables for Nuclear Power Plants"

2020 IEEE 3rd International Conference on Dielectrics (ICD), 2020, pp. 37-40

The final published version is available online at:

https://doi.org/10.1109/ICD46958.2020.9341856

Rights / License:

The terms and conditions for the reuse of this version of the manuscript are specified in the publishing policy. For all terms of use and more information see the publisher's website.

This item was downloaded from IRIS Università di Bologna (https://cris.unibo.it/)

When citing, please refer to the published version. 


\title{
Preliminary Development and Application of a Stress-Strength Model for Reliability Estimation of Aged LV Cables for Nuclear Power Plants
}

\author{
D. Fabiani, G. Mazzanti, S.V. Suraci, B. Diban \\ Department of Electrical Energy Engineering and Information "Guglielmo Marconi" \\ University of Bologna \\ Viale Risorgimento 2 \\ Bologna 40136, Italy
}

\begin{abstract}
The aim of this paper is to develop a model for the prediction of the residual reliability of Low Voltage $(\mathrm{LV})$ cables for Nuclear Power Plants (NPPs) subjected to gamma radiation stress. The model estimates the probability that such cables withstand random stress overshoot in-service.
\end{abstract}

\section{INTRODUCTION}

Low voltage (LV) cables have been widely used in Nuclear Power Plants (NPPs) for power transmission, control, instrumentation and communication. In service they are continuously subjected to a wide range of stresses which continuously degrade their insulation. Due to the catastrophic consequences of the aging-related accidents of LV cables which support control and safety measures in NPPs and due to the lifetime extension plans of current NPPs as well, the stresses applied to LV NPP cables have been extensively investigated [1]. This research is part of the H2020 EU Project called "TeaM Cables" which aims at providing NPP operators a novel methodology for efficient and reliable NPP cable ageing management. Among the aims of the Project, the modelling of the cable reliability over its application time is a key issue in order to efficiently schedule the cable replacement inside NPPs. Thus, an accurate model to predict the life and reliability of LV NPP cables in service is highly desirable. This paper is a first step for the development of a stress-strength model for the prediction of the residual reliability of LV NPP cables, based on the typical risk-assessment procedure of probabilistic insulation coordination.

\section{THEORETICAL AND EXPERIMENTAL BACKGROUND}

\section{A. Normal operation stresses}

Cable insulation aging in nuclear power plants is affected by both the dose of nuclear radiation absorbed by cable insulation over a certain aging time $t, D(t)$, and the temperature $T$ of cable insulation whose effect is also cumulated over aging time. This dose is cumulated over aging time at a certain dose rate $D R$ (radiation dose/time) which might vary over time. If the dose rate $D R$ is assumed as constant during the normal operation of the plant, the total dose $D(t)$ cumulated after a service time $t$ of the plant is equal to:

$$
D(t)=D R t
$$

It must be pointed out that nuclear radiation for the NPP LV cables under exam here essentially consists in gamma radiation.

During the nominal operation of the plant, dose rate $D R_{n}$ and temperature $T_{n}$ of cable insulation can be assumed as known and constant deterministic quantities, with the exception of anomalous events where stress overshoots may occur. $D R_{n}$ and $T_{n}$ are responsible for the irreversible degradation of cable insulation with time, which makes the electrical, thermal and mechanical properties of cable insulation worse over time with respect to their initial values (relevant to the unaged cable, or reached after a proper initial period of pretreatment/ conditioning/ training), until cable failure occurs. Thus, $D R_{n}$ and $T_{n}$ can be referred to as "stress factors".

One (or more) diagnostic property $P$ can associated with aging (e.g. electric strength, $E S$, dissipation factor, $\operatorname{tg} \delta$, tensile strength, $T S$, elongation at break, $E a B$ ) provided that these properties show a monotone behavior with aging time. Failure takes place when the selected property overcomes a critical limit, $P L$, also referred to as "end point". The selection of the diagnostic property $\mathrm{P}$ and of its end point $P L$ is referred to as "failure criterion".

Accordingly, the concept of dose equivalent to damage, $D E D$, can be defined as the value of cumulated dose that, if exceeded after service time $t$, gives rise to such a damage to cable insulation that the selected diagnostic property $P$ overcomes its end point $P_{L}$, namely: $E S \geq E S_{L}$, or $T S \geq T S_{L}$, or $E B \geq E B_{L}$, or $\operatorname{tg} \delta \geq(\operatorname{tg} \delta)_{L}$.

It must be emphasized that nuclear radiation and temperature are not per se destructive stresses, thus the selection of the end point is to some extent arbitrary.

\section{B. Stresses overshoots and Dose Equivalent to Damage}

Stresses overshoots may occur at aging time $t$, such that a burst of dose $D B$ is suddenly released, e.g. in case of a nuclear accident. In this respect, it is assumed that stress overshoots are random events, hence the release of a certain dose burst $D B$ in the presence of this phenomenon can be described by a proper probability distribution function, which has dose burst $D B$ as random variable: this random variable of stress is denoted as $\mathrm{X}$ in the framework of the probabilistic procedure hereafter. Therefore, if the sum of the dose cumulated so far at dose rate $D R_{n}$, eq.(1), and the dose $D B$ released as a burst during the 
overshoot is such that it exceeds $D E D$, then cable insulation will fail. This means that the hypothesized event occurring after a service time $t$ causes such a damage to cable insulation that the selected diagnostic property $\mathrm{P}(E a B$ and/or $\operatorname{tg} \delta)$ reaches its end point PL. This, by convention, corresponds to cable failure (end of life). Therefore, the critical condition for failure to occur can be written as follows, being $D B$ a random variable:

$$
D R t+D B \geq D E D
$$

Or alternatively as follows:

$$
D B \geq D E D-D R t
$$

Hence, in the framework of "stress-strength" models: the dose burst DB can be viewed as a sudden STRESS X; the residual dose that can be still cumulated by cable insulation at aging time $t$ before it fails, namely $D E D-D R t$, can be viewed as the residual STRENGTH Y at aging time $t$.

Actually, due to the random inhomogeneities of cable insulation, the dose equivalent to damage DED of cable insulation is not a deterministic quantity, but a random variable (rv) (like the other diagnostic properties, $E S, T S, E a B, \operatorname{tg} \delta$, etc.), as explained in detail later. The random variable DED makes the (residual) strength of the insulation of LV NPP cables a random variable as well. The random variable "strength $\mathrm{Y}$ at aging time t", equal to $D E D-D R t$ (see eq. (3) above), is denoted as $\mathrm{Y}(\mathrm{t})$ in the framework of probabilistic "StressStrength" models (see below) and the relationship between strength and service time $t$ is given by eq. (3). Then, the failure criterion expressed by (3) becomes:

$$
X=D B \geq Y(t)=D E D-D R t
$$

And conversely the withstand criterion in the framework of probabilistic "Stress-Strength" models becomes

$$
X=D B<Y(t)=D E D-D R t
$$

The cumulative distribution function $(c d f)$ of $D E D$ i.e. $P(D E D)$ is assumed to be a 2-parameter Weibull function which is well-suited for solid polymeric insulation, of the kind:

$$
P(D E D ; D R, T)=1-\exp \left[-\left(\frac{D E D}{\alpha_{D E D}(D R, T)}\right)^{\beta_{D E D}(D R, T)}\right]
$$

where $\alpha_{D E D}(D R, T)$ and $\beta_{D E D}(D R, T)$ are the scale and shape parameters of Weibull function and strictly speaking they depend on service stresses $D R$ and $T$, but in practice, $\beta_{D E D}$ can be taken as stress independent. Then, under the above assumption that during the nominal operation of the LV NPP cable insulation is subjected to known and constant nominal values of dose rate, $D R_{n}$, and temperature, $T_{n}$, equation (6) can be rewritten as follows:

$$
P\left(D E D ; D R_{n}, T_{n}\right)=1-\exp \left[-\left(\frac{D E D}{\alpha_{D E D}\left(D R_{n}, T_{n}\right)}\right)^{\beta_{D E D}}\right]
$$

C. Experimental Evaluation of Dose Equivalent to Damage

In the TEAMCABLES project, the first diagnostic property considered is $E a B$. The initial value of $E a B$ of the unaged cable is referred to as $e_{0}$, while the actual $E a B$ at a certain aging time is referred to as $e$. Three limit values of relative $E a B$ - calculated as $e / e_{0}$ - considered to be overcome for failure to take place are as follows:

- $40 \%$ of $e_{0}$. Then failure is said to take place as soon as relative $e / e_{0}=0.4$;

- $\quad 50 \%$ of $e_{0}$ (preferred criterion). Then failure is said to take place as soon as $e / e_{0}=0.5$;

- $60 \%$ of $e_{0}$. Then failure is said to take place as soon as $e / e_{0}=0.6$.

Graphs of $e / e_{0}$ vs cumulated dose $D$ have been obtained at three different dose rates [4]:

- $\quad$ small dose rate $\mathrm{SDR}=8.5 \mathrm{~Gy} / \mathrm{h}$;

- medium dose rate $\mathrm{MDR}=59 \mathrm{~Gy} / \mathrm{h}$;

- high dose rate $\mathrm{HDR}=400 \mathrm{~Gy} / \mathrm{h}$.

The graphs (see e.g. Fig. 1 after [4], relevant to HDR) show a sharp decrease of $e / e_{0}$ with $D$, as well as a dependence of the slope of the $e / e O$ vs. $D$ plot on dose rate $D R$, i.e. on aging conditions. Therefore, the results enabled the derivation of an empirical relationship between $D E D$ and $D R$, i.e.:

$$
D E D=K \cdot D R^{n}=167 \cdot D R^{0.16}(k G y)
$$

Subsequently, the value of $\operatorname{tg} \delta$ as a function of the total dose $D$ for the analyzed aging conditions was measured, so as to establish a connection between $E a B$ and $\operatorname{tg} \delta$ as prospective diagnostic properties [5]. This approach, which needs further investigations and analysis of bigger amount of data, would allow the definition of $\operatorname{tg} \delta$ end-of-life values for various kinds of cables and aging conditions.

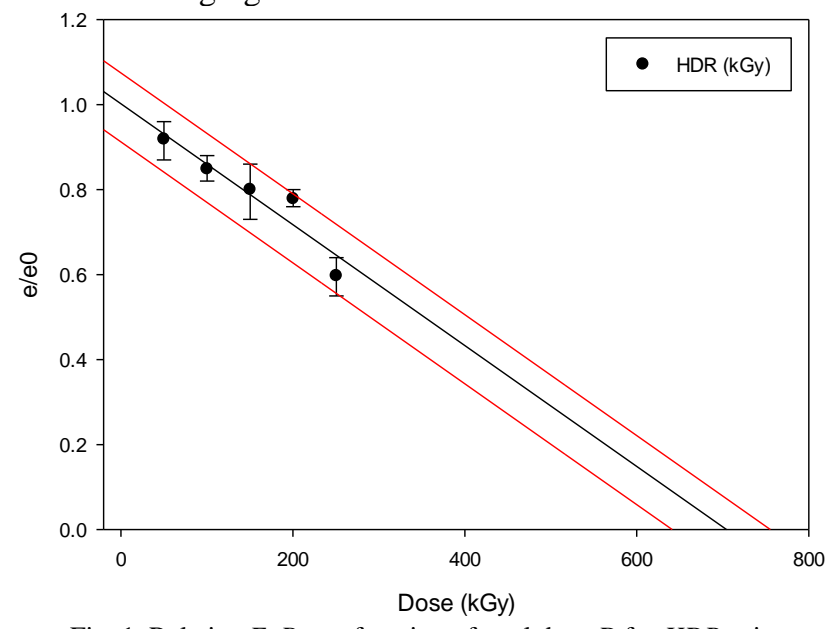

Fig. 1. Relative $E a B$ as a function of total dose $D$ for $H D R$ aging

\section{THE STRESS-STRENGTH RELIABILITY MODEL}

The reliability assessment problem of LV NPP cables is dealt with in the framework of probabilistic "Stress-Strength" models $[5,6]$, i.e. by writing the reliability function $(\mathrm{RF})$, for a given mission time $\mathrm{t}$, as:

$$
R=P(X<Y(t))
$$


where:

$X$ ("Stress") is dose burst DB released during the overshoot;

$Y(t)$ ("Strength" at aging time $\mathrm{t}$ ) is DED - DR.t.

Denoting with:

$f(y)=$ the probability density function (pdf) of Strength $Y$;

$F(y)=$ the cumulative distribution function (cdf) of Strength $Y$;

$g(x)=$ the $p d f$ of Stress $X$, namely dose burst $D B$;

$G(x)=$ the $c d f$ of Stress $X$;

Assuming, as generally accepted for the applications under study that the random variables $\mathrm{X}$ and $\mathrm{Y}$ are statistically independent, the RF of the LV cable is given by:

$$
\begin{array}{r}
R(t)=\int_{0}^{\infty} g(x) P(X<Y(t) \mid X=x) d x= \\
\quad=\int_{0}^{\infty} g(x)\{1-F[x(t)]\} d x= \\
=\int_{0}^{\infty} g(x)\{R[x(t)]\} d x
\end{array}
$$

From the above hypotheses, $F[Y(t)]$, the cdf of strength at time $t$, is not coincident with the cdf of $D E D$ - this latter being a Weibull cdf (see eqns. (6),(7)), with scale and shape parameter, $\alpha_{D E D}\left(D R_{n}, T_{n}\right)$ and $\beta_{D E D}$.

However, at a certain aging time t, it holds (see eq. (4)):

$$
\begin{aligned}
Y(t) & =D E D-D R t \\
F[y(t)] & =F[D E D-D R t]
\end{aligned}
$$

where - at a fixed aging time $t-D E D$ is a random variable distributed according to (7) and DR.t is a constant deterministic quantity. Thus, $Y(t)$ at a fixed aging time $t$ will also be Weibulldistributed according to (7), on condition that the $c d f$ is shifted along the random variable axis by an amount + DR.t so as to compensate for the reduction of $\mathrm{Y}(\mathrm{t})$ with respect to its initial value $D E D$. Thus, it holds:

$$
\begin{aligned}
& F[y(t)]=1-\exp \left[-\left(\frac{D E D+D R \cdot t}{\alpha_{D E D}}\right)^{\beta_{D E D}}\right]= \\
& =1-\exp \left[-\left(\frac{D E D}{\alpha_{D E D}}\right)^{\beta_{D E D}}\right] \exp \left[-\left(\frac{D R t}{\alpha_{D E D}}\right)^{\beta_{D E D}}\right]
\end{aligned}
$$

which shows how the time variable can be decoupled from the strength variable.

An example of the $c d f$ of $Y(t)$ according to (13) is given in Fig. 2, which shows the Weibull $c d f$ of strength $\mathrm{Y}(\mathrm{t})$ with $\alpha_{D E D}\left(D R_{n}, T_{n}\right)=350 k G y \quad$ (see $\quad$ Fig. 2$), \quad \beta_{D E D}=10$ (parametrically assumed values) at three different times:

- $t=0$, corresponding to initial strength $(Y(0)=D E D$, see eq. (11));

- $t=t_{1}$, corresponding to $70 \%$ of initial strength;

- $t=t_{2}$, corresponding to, $43 \%$ of initial strength.

The mathematical model for the evaluation of the RF of eq. (10) is generally quite difficult (or impossible) to be solved analytically, except for a few cases. Here, a very simple model is chosen, namely the one in which also stress $\mathrm{X}$ is a Weibull rv. Thus, the pdf of stress, $\mathrm{g}(x)$, in (10) can be derived from the following cdf of stress:

$$
G(x)=1-\exp \left[-(x / \theta)^{\gamma}\right]
$$

whose scale and shape parameters are $\theta$ and $\gamma$, respectively. As a further simplification, let us assume:

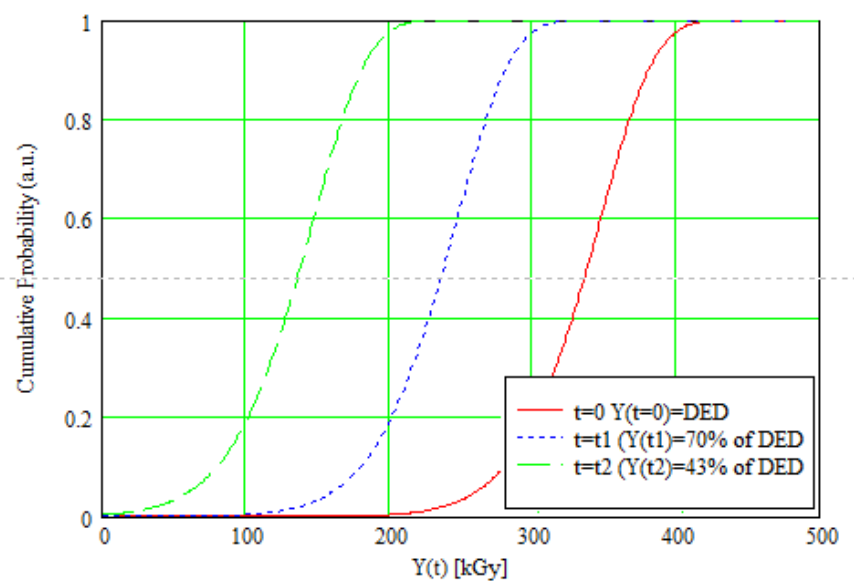

Fig. 2. cdf of strength $Y(t)$ at three different times corresponding to initial strength (=DED), $70 \%$ of initial strength, $43 \%$ of initial strength

$$
\gamma=\beta
$$

These assumptions are justified here, since the first purpose of this investigation is to propose a new estimation methodology applied to LV cables for nuclear power plants for the first time in its simplest version. Moreover, observed stress distributions in some cases are not far from eq. (14).

\section{CASE-STUDY}

As a preliminary parametric case-study, selected for the sake of illustration of the proposed stress-strength reliability model for aged NPP LV cables, let us apply eq. (10) with the following values of the relevant parameters:

- $\alpha=350 \mathrm{kGy}, \beta=\gamma=10$ (see Fig. 2);

- $\theta=0.5 \alpha, 0.75 \alpha, 0.875 \alpha, \alpha$, so as to scan a range of possible values of shape parameter of the stress distribution and assess the relevant effects;

- $D R=\mathrm{SDR}=8.5 \mathrm{~Gy} / \mathrm{h}, \mathrm{MDR}=59 \mathrm{~Gy} / \mathrm{h}, \mathrm{HDR}=400 \mathrm{~Gy} / \mathrm{h}$ after [4]

The values of reliability $R(t)$ vs. aging time $t$ for SDR and HDR are shown, respectively, in Figs. 3(a), 3(b).

The figures clearly show that, as reasonably expected:

- the higher the dose rate, the lower the reliability at a given time for each value of stress scale parameter, $\theta$;

- the higher the scale parameter of stress, $\theta$, the lower the reliability at a given time and value of dose rate.

Another point deserves attention. Since $\theta=0.75 \alpha$ initial reliability $R(t=0)$ starts being $<1$, and the higher $\theta$, the more $R(t=0)$ is below unity. This stems from the fact that stress is a dose burst at a given time $t$, and reliability $R(t)$ is the likelihood that cable insulation withstands that dose burst distributed 
according to eq. (14). Of course, the higher is $\theta$ the more the cdf $G(x) /$ pdf $g(x)$ of stress is displaced towards higher values of dose bursts, hence the more likely is that the insulation is not capable of withstanding the stress at a given time $t$, including $t=0$. Indeed, according to insulation coordination theory, the risk of such event, $\operatorname{Risk}(t=0)$ is the convolution of strength cdf $F[y(t=0)]=F[x(t=0)]$ and stress pdf $g(x)$, i.e. (see also eq.(10)):

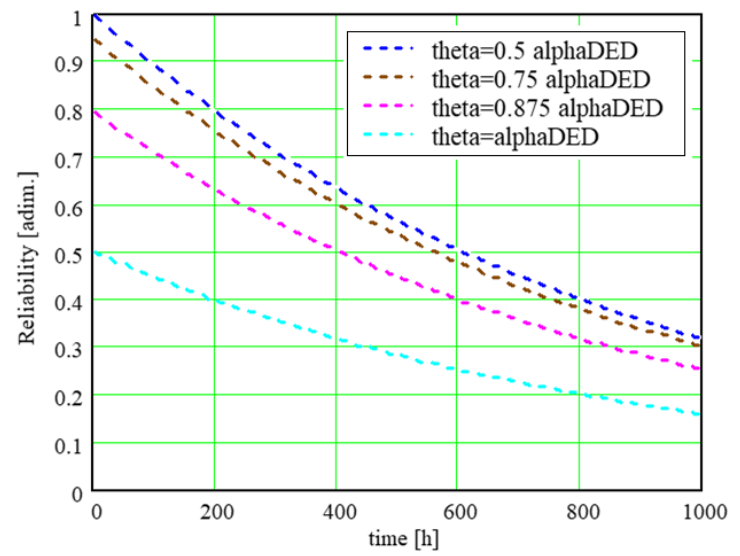

(a)

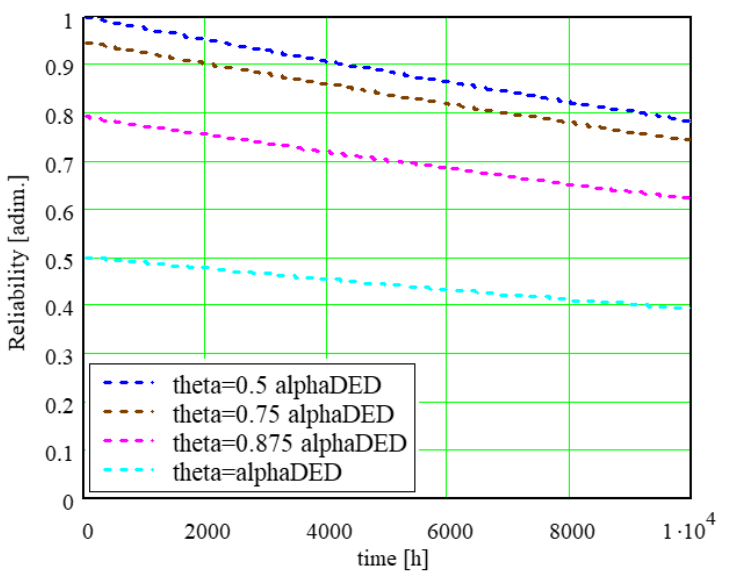

(b)

Fig. 3. Reliability according to eq. (25) for (a) HDR and (b) LDR, at various values of $\theta$. Function $\zeta(t)$ is also shown.

$$
\operatorname{Risk}(t=0)=\int_{0}^{\infty} g(x) F[x(t=0)] d x
$$

and may be $\neq 0$, as it can be argued from Fig. 4 for HDR.

Figure 4 - zoomed on the ordinate for the sake of clarity shows the strength cdf $F[y(t=0)]$ and the various stress pdfs $g 0(x), g 1(x), g 2(x), g 3(x)$, obtained respectively for $\theta=0.5 \alpha$, $0.75 \alpha, 0.875 \alpha, \alpha$. It is readily seen that for $\theta=0.5 \alpha, g(x)$ is totally to the left of strength curve cdf $F[y(t=0)]$, thus $\operatorname{Risk}(t=0)$ is expected to be zero according to eq. (16). On the contrary, since $\theta=0.75 \alpha, g(x)$ starts being displaced to the right of strength curve, and the higher $\theta$, the more $g(x)$ is displaced to the right, thereby raising $\operatorname{Risk}(t=0)$, thus reducing $R(t=0)$

\section{CONCLUSION}

In this article, a preliminary development and application of a model of residual reliability of LV cables for NPPs has been presented. This model, based on the stress-strength reliability model theory, allowed the prediction of the probability that such cables withstand random stress overshoots in-service. In this initial stage a number of assumptions has been made in order to obtain an estimation methodology applied to LV cables in its simplest version. Moreover, a parametrical case-study has been reported and showed an accurate reflection of the behavior of real cables already in use inside NPPs.

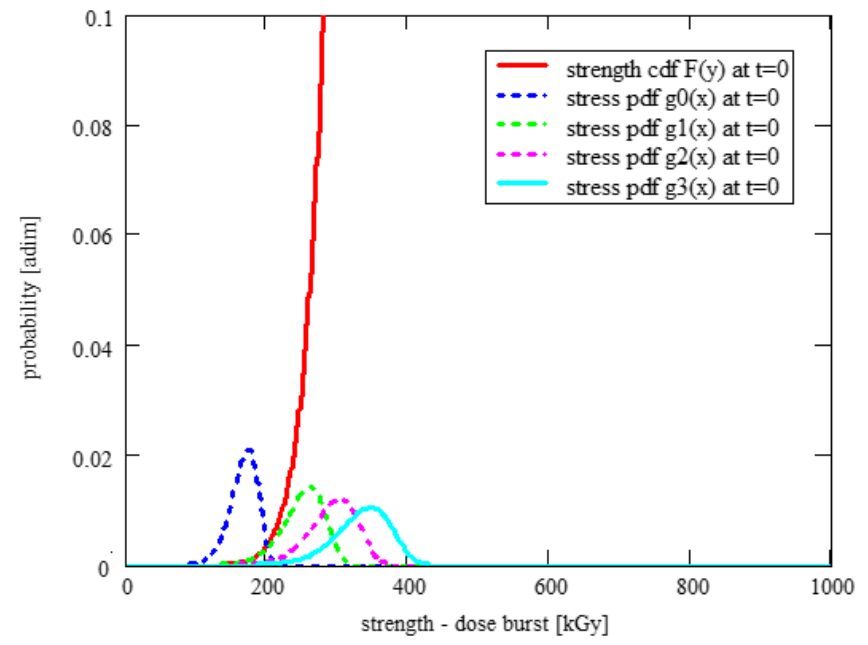

Figure 4. Strength cdf $F[y(t=0)]$ and the various stress pdfs $g 0(x), g 1(x), g 2(x)$, $g 3(x)$, obtained respectively for $\theta=0.5 \alpha, 0.75 \alpha, 0.875 \alpha, \alpha$.

Future work on this topic will include a further investigation of the theoretical approach proposed together with a deeper parametric analysis of the different variables. Furthermore, the application of the same method will be used in other cables with different additives composition and morphologies in order to investigate the impact of these parameters on the given modelling approach.

\section{ACKNOWLEDGMENT}

The project leading to this application has received funding from the

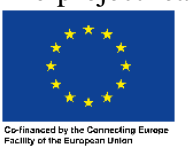
Euratom research and training programme 2014-2018 under grant agreement No 755183. The authors are grateful to Nexans and UJV for sample preparation and aging.

\section{REFERENCES}

[1] L. Verardi, D. Fabiani, G. C. Montanari, V. Placek, "Electrical aging markers for low-voltage cable insulation wiring of nuclear power plants", Con! proc. of IEEE Int. Conference on Properties and Application of Dielectric Materials (ICPADM), pp. 1-4.

[2] E. Chiodo, D. Fabiani, G. Mazzanti, "Bayes Inference for Reliability of HV Insulation Systems in the Presence of Switching Voltage Surges Using a Weibull Stress-Strength Model", 2003 IEEE Bologna Power Tech Conference, Bologna, 22-26 June 2003, ISBN 0-7803-7967-5/03 (article), ISBN 0-7803-7968-3.

[3] E. Chiodo, G. Mazzanti, "Bayesian reliability estimation based on a Weibull stress-strength model for aged power system components subjected to voltage surges", IEEE Transactions on Dielectrics and Electrical Insulation, Vol.13, n. 1, pp. 146-159, February 2006.

[4] S.V. Suraci, D. Fabiani, Z. Sarsounova, P. Zak, "Life modeling analysis of Silane crosslinked polyethylene cables for nuclear applications", 2020 IEEE ICD Valencia.

[5] S. V. Suraci, D. Fabiani, A. Xu, S. Roland, and X. Colin, "Ageing Assessment of XLPE LV Cables for Nuclear Applications Through Physico-Chemical and Electrical Measurements," IEEE Access, vol. 8, pp. 27086-27096, 2020, doi: 10.1109/ACCESS.2020.2970833. 